lapses of time occur; the characters remain the same age; they are all contemporaries; supernatural beings are always personified; shapechanging is common; kings and queens always appear; battles consist of single combats; prophecies always come true; there is usually a character who directs the action.

The belief in the historicity of the traditional narrative is a survival from the pre-scientific age, one of a number of such survivals the existence of which prevents social anthropology from taking what should be its proper place among the sciences.

\section{Activities of Nerve Cells}

IN his presidential address to Section I (Physiology), Prof. E. D. Adrian discusses the central nervous system and the prospect of giving a satisfactory explanation of its activities. To the majority of physiologists a satisfactory explanation must be one which shows how the individual cells of the nervous system combine to direct the behaviour of the organism.

From one point of view the prospects are encouraging. The neurones, or units of the nervous system, are built up on an elaborate plan, but experimental embryology has begun to determine the forces which mould the developing cells and direct the long threads of protoplasm which grow out from them to form the nerve fibres. Work on other lines has shown that in their main reactions the neurones do not differ greatly from other kinds of excitable cell. The rapid transmission of signals over long distances is carried out in a simple way by trains of brief impulses passing along the nerve fibres. The impulses are set up in the sense organs and other specialised parts of the neurone, but similar trains of impulses can be set up in muscle fibres and their production seems to depend in both cases on the same kind of rhythmic breakdown and repair of a polarised surface.

Recent investigations of the electric changes in the cerebral cortex and in nerve ganglia show that in the grey matter these surface changes may have much slower and less uniform time relations. There are, however, many intermediate stages between the rhythmic succession of impulses in a nerve fibre and the slower and more irregular oscillations in the brain cortex.

The difficulties begin when we turn from the units to consider the nervous system as a whole. The simple diagrams of nerve centres and of path- ways canalised by use can no longer be accepted. They have failed to explain the facts of habit formation and the rapid return of function after injury. Localisation of function certainly exists in the brain, but it is a matter of large areas rather than of small cell groups. A particular relation of sensory stimuli leads to a particular movement of the organism, and the relation is recognised and the movement achieved without much regard to the nerve tracts employed in forming the association. The importance, in learning, of the total mass of the cortex and the unimportance of particular regions is another example of this unified action of the brain.

To account for it should not be beyond the range of experiment, for there are many cases in which it is possible to study the action of smaller groups of nerve cells. The electric changes taking place in such groups and in the cerebral cortex show that the different neurones often pulsate synchronously, as though they formed a mass through which the waves of activity are freely conducted. In such a mass the waves due to incoming signals and to the spontaneous activity of nerve cells may produce nodes of vibration and interference figures, patterns in time as well as in space. Such patterns might be independent of precise neurone connexions. It is possible that they may supply the basis for an appropriate motor response, though how they can do so is a problem to which no answer can be given in the present state of our knowledge.

At the moment no one would claim that we have a satisfactory picture of the mechanisms at work in the brain. It may be that such a picture is unattainable ; but at least we have new problems and the prospect of fresh data in a most interesting field.

\section{Empirical Basis of Psychology}

TN his presidential address to Section $J$ (Psychology), entitled "The Status of Psychology as an Empirical Science", Prof. F. Aveling develops the theme that psychology is the most radically empirical of all the sciences because it is concerned with the totality of immediate experience rather than with remote inferences from selected parts of sensory experience, as are all the other natural sciences.

To establish this contention, a distinction is first drawn between several meanings of the term 'empirical'. A science is said to be empirical if 